\title{
SIMPLE $(-1,-1)$ BALANCED FREUDENTHAL KANTOR TRIPLE SYSTEMS
}

\author{
ALBERTO ELDUQUE* \\ Departamento de Matemáticas, Facultad de Ciencias, Universidad de Zaragoza, 50009 Zaragoza, Spain \\ e-mail: elduque@unizar.es \\ NORIAKI KAMIYA** \\ Center for Mathematical Sciences, The University of Aizu, 965-8580 Aizu-Wakamatsu, Japan \\ e-mail:kamiya@u-aizu.ac.jp \\ and SUSUMU OKUBO*** \\ Department of Physics and Astronomy, University of Rochester, Rochester, NY 14627, USA \\ e-mail: okubo@pas.rochester.edu
}

(Received 8 April, 2002; accepted 8 November, 2002)

\begin{abstract}
The simple finite dimensional $(-1,-1)$ balanced Freudenthal Kantor triple systems over fields of characteristic zero are classified.
\end{abstract}

2000 Mathematics Subject Classification. 17A40, 17B20, 17B25.

1. Introduction. In 1954, H. Freudenthal [10] constructed the exceptional simple Lie algebras of types $E_{7}$ and $E_{8}$ by means of the exceptional simple Jordan algebras. The construction of $E_{8}$ has been extended in several ways to give 5-graded Lie algebras

$$
\mathfrak{g}=\mathfrak{g}_{-2} \oplus \mathfrak{g}_{-1} \oplus \mathfrak{g}_{0} \oplus \mathfrak{g}_{1} \oplus \mathfrak{g}_{2}
$$

starting with some nonassociative algebras or triple systems, which appear as the component $\mathfrak{g}_{1}$.

The concept of $(\varepsilon, \delta)$-Freudenthal Kantor triple system covers many of these systems:

Definition 1.1 [29]. Let $\varepsilon, \delta= \pm 1$. A vector space $V$ over a field $F$, endowed with a trilinear operation $V \times V \times V \rightarrow V,(x, y, z) \mapsto x y z$, is said to be a $(\varepsilon, \delta)$-Freudenthal Kantor triple system $((\varepsilon, \delta)$-FKTS for short) if the following two conditions are satisfied

(i) $\left[l_{a, b}, l_{c, d}\right]=l_{l, b} c, d+\varepsilon l_{c, l_{b, a} d}$,

(ii) $l_{d, c} k_{a, b}-\varepsilon k_{a, b} l_{c, d}=k_{k_{a, b} c, d}$

for any $a, b, c, d \in V$, where $l_{a, b}, k_{a, b}: V \rightarrow V$ are given by $l_{a, b} c=a b c, k_{a, b} c=a c b-$ Sbca.

Thus a $(-1,1)$-FKTS is exactly a generalized Jordan triple system of second order in the sense of Kantor [20] (if $k=0$ this is just a Jordan triple system), while a

\footnotetext{
*Supported by the Spanish MCYT (BFM 2001-3239-C03-03).

** Supported in part by a Ministry of Education of Japan grant (no. 10640024).

*** Supported in part by U.S. Department of Energy Grant no. DE-F402-ER 40685.
} 
$(1,-1)$-FKTS with $k=0$ is an anti-Jordan triple system (see [9] for the definition of anti-Jordan pair $\left(U^{+}, U^{-}\right)$; when $U^{+}=U^{-}$one gets an anti-Jordan triple system).

An $(\varepsilon, \delta)$-FKTS $V$ is said to be balanced $((\varepsilon, \delta)$-BFKTS for short) if there exists a nonzero bilinear form (|) $: V \times V \rightarrow F$ such that $k_{a, b}=(a \mid b) 1_{V}$ for any $a, b \in V\left(1_{V}\right.$ denotes the identity map on $V$ ). Since $k_{a, b}=-\delta k_{b, a}$ by its own definition, ( $\mid$ ) is either symmetric $(\delta=-1)$ or skew-symmetric $(\delta=1)$. On the other hand, condition (ii) in Definition 1.1 gives here that $(\mid)$ is either symmetric or skew-symmetric according to $\varepsilon$ being -1 or 1 , so that $\varepsilon=\delta$ in case $V$ is balanced.

Any $(1,1)$-BFKTS becomes, by means of minor modifications of its triple product, a symplectic ternary algebra [8], a symplectic triple system [28] or a Freudenthal triple system [21], and conversely. The simple finite dimensional Freudenthal triple systems were classified in [21], with some restrictions which are satisfied if the ground field is algebraically closed, and this amounts to a classification of the simple $(1,1)$-BFKTS (and of the symplectic ternary algebras [8]). The related 5-graded Lie algebras satisfy that $\mathfrak{g}_{ \pm 2}$ is one dimensional.

Further properties of $(\varepsilon, \delta)$-FKTS's can be found in $[\mathbf{1 2 - 1 8}, \mathbf{2 4}]$ and the references therein.

Our aim in this paper is to obtain the classification of the finite dimensional simple $(-1,-1)$-BFKTS's over fields of characteristic 0 . To achieve this, the classification [11] of the finite dimensional simple Lie superalgebras over algebraically closed fields of characteristic 0 will be used, but we will have to look at the known relationship between $(-1,-1)$-FKTS's and 5-graded Lie superalgebras [27] in a different way, suitable to our needs. This will be done in Section 2. The relevant examples of $(-1,-1)$-BFKTS's will be given in Section 3 and, finally, Section 4 will provide the promised classification (Theorem 4.3), which asserts that the simple finite dimensional $(-1,-1)$-BFKTS's fall into six classes, three of them with arbitrarily large dimension: orthogonal, unitarian and symplectic types; and another three classes of four dimensional ( $\mathrm{D}_{\mu}$-type), seven dimensional (G-type) and eight dimensional systems (F-type).

Using Definition 1.1, the defining relations for a $(-1,-1)$-BFKTS are

$$
\begin{gathered}
a b(x y z)=(a b x) y z-x(b a y) z+x y(a b z), \\
a b x+b a x=(a \mid b) x=a x b+b x a,
\end{gathered}
$$

for any $a, b, x, y, z \in V$, where ( $\mid$ ) is a nonzero symmetric bilinear form. Over fields of characteristic $\neq 2$, put $\langle\mid\rangle=\frac{1}{2}(\mid)$ and then (1.2) is equivalent to

$$
x x y=\langle x \mid x\rangle y=x y x
$$

for any $x, y \in V$.

The main motivation for the classification of the simple $(-1,-1)$-BFKTS's was provided by the recent paper [19] by two of the authors, where the exceptional simple classical Lie superalgebras were constructed by using the last three classes mentioned above (D, G and $\mathrm{F}$ types). These triple systems are closely related to quaternion and octonion algebras. A different construction of the exceptional simple classical Lie superalgebras has been given in [2] by means of a generalized Tits' construction (which also uses quaternion and octonion algebras).

2. (-1, -1) balanced Freudenthal Kantor triple systems and Lie superalgebras. The relationship between $(-1,-1)$-BFKTS and Lie superalgebras has been studied in [19]. A more useful approach for us is obtained as indicated by the next Theorem. 
THEOREM 2.1. Let $\mathfrak{g}$ be a finite dimensional Lie superalgebra over a field $F$ of characteristic $\neq 2$ such that $\mathfrak{g}_{\overline{0}}=\mathfrak{s l}_{2}(F) \oplus \mathfrak{d}$ (direct sum of ideals) and $\mathfrak{g}_{\overline{1}}=U \otimes_{F} V$, where $U$ is the two dimensional module for $\mathfrak{s l}_{2}(F)$ and $V$ is a module for $\mathfrak{d}$. Let $\varphi$ be a nonzero skew symmetric form on $U$, so that we may identify $\mathfrak{s l}_{2}(F)=\mathfrak{s p}(U, \varphi)$ and for any $a, b \in U$ consider the map $\varphi_{a, b} \in \mathfrak{s l}_{2}(F)$ given by

$$
\varphi_{a, b}(c)=\varphi(c, a) b+\varphi(c, b) a
$$

for any $c \in U$. Then the product of odd elements in $\mathfrak{g}$ is given by

$$
[a \otimes u, b \otimes v]=\langle u \mid v\rangle \varphi_{a, b}+\varphi(a, b) d_{u, v}
$$

for any $a, b \in U$ and $u, v \in V$, where $\langle\mid\rangle$ is a symmetric bilinear form and $V \times V \rightarrow \mathfrak{d}$, $(x, y) \mapsto d_{x, y}$, is a skew symmetric bilinear map that satisfy

$$
\begin{gathered}
\langle d(x) \mid y\rangle+\langle x \mid d(y)\rangle=0, \\
{\left[d, d_{x, y}\right]=d_{d(x), y}+d_{x, d(y)},} \\
d_{x, y}(y)=\langle y \mid x\rangle y-\langle y \mid y\rangle x,
\end{gathered}
$$

for any $x, y \in V$ and $d \in \mathfrak{d}$.

Conversely, let $V$ be a vector space endowed with a symmetric bilinear form $\langle\mid\rangle$ : $V \times V \rightarrow F$ and a skew symmetric bilinear map $V \times V \rightarrow \operatorname{End}_{F}(V)\left((u, v) \mapsto d_{u, v}\right)$. Assume that:

$$
\begin{array}{r}
\left\langle d_{u, v}(x) \mid y\right\rangle+\left\langle x \mid d_{u, v}(y)\right\rangle=0, \\
{\left[d_{u, v}, d_{x, y}\right]=d_{d_{u, v}(x), y}+d_{x, d_{u, v}(y),}} \\
d_{x, y}(y)=\langle y \mid x\rangle y-\langle y \mid y\rangle x,
\end{array}
$$

for any $u, v, x, y \in V$. Let $\mathfrak{d}$ be $\operatorname{span}\left\{d_{u, v}: u, v \in V\right\}$ (a Lie subalgebra of $\operatorname{End}_{F}(V)$ by $(2.3 b)$ ) and let $\mathfrak{g}=\mathfrak{g}_{\overline{0}} \oplus \mathfrak{g}_{\overline{1}}$ be the superalgebra where $\mathfrak{g}_{0}$ is the Lie algebra $\mathfrak{s l}_{2}(F) \oplus \mathfrak{d}=$ $\mathfrak{s p}(U, \varphi) \oplus \mathfrak{d}, \mathfrak{g}_{\overline{1}}$ is the $\mathfrak{g}_{0}$-module $U \otimes_{F} V$ and where the product of odd elements is given by (2.1). Then $\mathfrak{g}$ is a Lie superalgebra.

Proof. Since $\operatorname{Hom}_{\mathfrak{s p}(U, \varphi)}\left(U \otimes_{F} U, F\right)$ is spanned by the form $\varphi$ and $\operatorname{Hom}_{\mathfrak{s p}(U, \varphi)}\left(U \otimes_{F} U, \mathfrak{s p}(U, \varphi)\right)$ is spanned by the symmetric map $a \otimes b \mapsto \varphi_{a, b}$, formula (2.1) follows. Formulae (2.2a) and (2.2b) follow from the Jacobi superidentity applied to the elements $d \in \mathfrak{d}$ and $a \otimes x, b \otimes y \in U \otimes_{F} V$ and (2.2c) follows from the Jacobi superidentity applied to three odd elements.

The converse is a straightforward computation.

With $V, V \times V \rightarrow \operatorname{End}_{F}(V),(x, y) \mapsto d_{x, y}$, and $\langle\mid\rangle$ as before, consider the triple product in $V$ given by

$$
x y z=d_{x, y} z+\langle x \mid y\rangle z
$$

for any $x, y, z \in V$. Conditions $(2.3 \mathrm{a}-\mathrm{c})$ translate into

$$
\begin{gathered}
x x y=\langle x \mid x\rangle y=x y x \\
u v(x y z)=(u v x) y z-x(v u y) z+x y(u v z), \\
\langle u v x \mid y\rangle=\langle x \mid v u y\rangle
\end{gathered}
$$


for any $u, v, x, y, z \in V$. Let us check (2.5b) for instance. For this, denote by $l_{x, y}$ the map $z \mapsto x y z$ for any $x, y, z \in V$, then for any $u, v, x, y \in V$

$$
\begin{aligned}
{\left[l_{u, v}, l_{x, y}\right] } & =\left[d_{u, v}, d_{x, y}\right] \quad \text { (since } l_{u, v}-d_{u, v} \text { is scalar) } \\
& =d_{d_{u, v}(x), y}+d_{x, d_{u, v}(y)} \\
& =l_{d_{u, v}(x), y}-\left\langle d_{u, v}(x) \mid y\right\rangle+l_{x, d_{u, v}(y)}-\left\langle x \mid d_{u, v}(y)\right\rangle \\
& =l_{d_{u, v}(x), y}-l_{x, d_{v, u}(y)} \\
& =l_{u v x, y}-\langle u \mid v\rangle l_{x, y}-l_{x, v u y}+\langle v \mid u\rangle l_{x, y} \\
& =l_{u v x, y}-l_{x, v u y}
\end{aligned}
$$

and this is equivalent to (2.5b). Conversely, conditions $(2.5 \mathrm{a}-\mathrm{c})$ give conditions $(2.3 \mathrm{a}-\mathrm{c})$, if (2.4) is used to define $d_{x, y}$ for $x, y \in V$.

Conditions (2.5a) and (2.5b) are just the defining conditions (1.3) and (1.1) of a $(-1,-1)$-BFKTS, while condition $(2.5 \mathrm{c})$ is a consequence of $(2.5 \mathrm{a}-\mathrm{b})$ [13]. We include a proof of this fact by completeness:

Take $x=y$ in (2.5b) and use (2.5a) to get

$$
\begin{aligned}
\langle x \mid x\rangle u v z & =(u v x) x z-x(v u x) z+\langle x \mid x\rangle u v z \\
& =(u v x) x z+((v u x) x z-2\langle x \mid v u x\rangle z)+\langle x \mid x\rangle u v z \\
& =2\langle u \mid v\rangle x x z-2\langle x \mid v u x\rangle z+\langle x \mid x\rangle u v z
\end{aligned}
$$

and this shows that $\langle x \mid v u x\rangle=\langle u \mid v\rangle\langle x \mid x\rangle$ for any $x, u, v \in V$. Linearizing this one obtains that $\langle x \mid v u y\rangle+\langle y \mid v u x\rangle=2\langle u \mid v\rangle\langle x \mid y\rangle$ for any $x, y, u, v \in V$, whence

$$
\langle x \mid v u y\rangle=\langle 2\langle u \mid v\rangle x-v u x \mid y\rangle=\langle u v x \mid y\rangle,
$$

as desired. In the same way, (2.3a) follows from (2.3b) and (2.3c).

Because of $(2.3 \mathrm{a}-\mathrm{b}), \mathfrak{d}=d_{V, V}$ is a Lie algebra of derivations of the $(-1,-1)$ BFKTS, which will be said to be the Lie algebra of inner derivations of $V$.

Given a vector space $V$ endowed with a nonzero symmetric bilinear form $\langle\mid\rangle$ and a skew symmetric map $V \times V \rightarrow \operatorname{End}_{F}(V),(x, y) \mapsto d_{x, y}$ for any $x, y \in V$, satisfying conditions (2.3), denote by $\mathfrak{g}(V)$ the Lie superalgebra constructed in Theorem 2.1. Also, consider the triple product $x y z$ defined on $V$ by (2.4) and the triple product given by $\{x y z\}=d_{x, y}(z)$ for any $x, y, z \in V$.

THEOREM 2.2. Under the hypotheses above, the following conditions are equivalent:

(i) $\langle\mid\rangle$ is nondegenerate,

(ii) $(V,\{x y z\})$ is a simple triple system,

(iii) $(V, x y z)$ is a simple triple system,

(iv) $\mathfrak{g}(V)$ is a simple Lie superalgebra.

Proof. Assume that (i) is satisfied and let $I$ be a nonzero ideal of the triple system $(V,\{x y z\})$. Then for any $x \in I$ and $y \in V,\{x y y\}=d_{x, y}(y)=-\langle y \mid y\rangle x+\langle x \mid y\rangle y \in I$, by (2.3c), and hence $\langle x \mid y\rangle y \in I$ for any $y \in V$. Since $\langle\mid\rangle$ is nondegenerate, there is a basis of $V$ formed by elements $y$ with $\langle x \mid y\rangle \neq 0$ and this shows that $I=V$. Conversely, $V^{\perp}=\{x \in V:\langle x \mid V\rangle=0\}$ is an ideal of $(V,\{x y z\})$ because of (2.3a) and the linearization of (2.3c). Hence (ii) implies (i).

Similarly, condition (i) and the linearization of (2.5a) imply (iii), and conversely (iii) implies (i) since $V^{\perp}$ is an ideal of $(V, x y z)$ because of (2.5a) and (2.5c). 
Now assume that (i) is satisfied and that $0 \neq I=I_{\overline{0}} \oplus I_{\overline{1}}$ is an ideal of the Lie superalgebra $\mathfrak{g}(V)$. By $\mathfrak{s l}_{2}(F)$-invariance, $I_{\overline{1}}=U \otimes_{F} W$ for a subspace $W$ of $V$. Let $x \in V$ and $y \in W$ with $\langle x \mid y\rangle \neq 0$, then for any $a \in U,[a \otimes x, a \otimes y]=-\langle x \mid y\rangle \varphi_{a, a}$, so $\varphi_{a, a} \in I_{\overline{0}}$ for any $a$ and $\mathfrak{s l}_{2}(F) \subseteq I_{\overline{0}}$. But then $\mathfrak{g}_{\overline{1}}=\left[\mathfrak{s l}_{2}(F), \mathfrak{g}_{\overline{1}}\right] \subseteq I$ and $\mathfrak{g}_{\overline{0}}=\left[\mathfrak{g}_{\overline{1}}, \mathfrak{g}_{\overline{1}}\right] \subseteq I$, so $I=\mathfrak{g}$. Otherwise $W=0$, so $I_{\overline{1}}=0$, but then it is easy to show that $I=0$.

Conversely, the graded subspace $d_{V, V^{\perp}} \oplus\left(U \otimes_{F} V^{\perp}\right)$ is an ideal of $\mathfrak{g}(V)$, so (iv) implies (i).

Since the nondegeneracy of a bilinear form is preserved under scalar extensions, it immediately follows that:

COROLlary 2.3. With the same notation as above, if $\langle\mid\rangle$ is nondegenerate, then $(V,\{x y z\}),(V, x y z)$ and $\mathfrak{g}(V)$ are central simple.

3. Examples. This section is devoted to constructing the examples of simple $(-1,-1)$ balanced Freudenthal Kantor triple systems that will appear in the classification. Throughout this section, the ground field $F$ will be assumed of characteristic $\neq 2$.

3.1. Hermitian type. Let $R$ be a unital separable associative algebra over $F$ of degree $\leq 2$. Therefore, $R$ is, up to isomorphism, either the ground field $F, F \times F$, a quadratic separable field extension $K$ of $F$ or a quaternion algebra $Q$ over $F$. In any case, $R$ is endowed with an involution of the first kind, $x \mapsto \bar{x}$, such that $x+\bar{x}, x \bar{x}=\bar{x} x \in F$ for any $x \in R$. Let $V$ be a left module over $R$ endowed with a nondegenerate hermitian form $h: V \times V \rightarrow R$. That is, $h$ is $F$-bilinear and satisfies for any $x, y \in V$ and $r \in R$ :

$$
\begin{aligned}
h(r x, y) & =r h(x, y), \\
h(x, y) & =\overline{h(y, x)}, \\
h(x, V) & =0 \text { if and only if } x=0 .
\end{aligned}
$$

Then the symmetric bilinear form $V \times V \rightarrow F$ defined by means of

$$
\langle x \mid y\rangle=\frac{1}{2}(h(x, y)+h(y, x)),
$$

for any $x, y \in V$, is nondegenerate and determines $h$.

Define now the triple product on $V$ by means of

$$
x y z=h(z, x) y-h(z, y) x+h(x, y) z,
$$

for any $x, y, z \in V$.

It is clear that $x x y=h(x, x) y=\langle x \mid x\rangle y=x y x$ for any $x, y \in V$ and a straightforward computation shows that this triple product satisfies $(2.5 \mathrm{~b})$ too. Therefore $V$ is a $(-1,-1)$-BFKTS which will be said to be of hermitian type. Depending on $\operatorname{dim}_{F} R$ being either 1, 2 or 4, $V$ will be said to be of orthogonal, unitarian or symplectic type, respectively, for reasons that will become clear later on.

Let us compute the Lie algebra $\mathfrak{d}=d_{V, V}$ in this case. Assume first that $R=F$, the ground field, then $d_{x, y}=\langle-\mid x\rangle y-\langle-\mid y\rangle x=: \sigma_{x, y}$ for any $x, y \in V$, and these maps span the orthogonal Lie algebra o $(V)$. From the construction in [11, Supplement to 2.1.2], $\mathfrak{g}(V)$ is the orthosymplectic Lie superalgebra $\mathfrak{o s p}(V \oplus U)$. A word of caution 
is needed here: the multiplication of odd elements in [11, Supplement to 2.1.2] should $\operatorname{read}[a \otimes c, b \otimes d]=-(a, b)_{0} c \circ d+(c, d)_{1} a \wedge b$ (a minus sign has been added).

Now, in case $R$ is a quadratic étale algebra, that is, either $K=F \times F$ or $K$ is a quadratic field extension of $F$, then for any $x, y \in V$,

$$
d_{x, y}=h_{x, y}+h_{0}(x, y) 1_{V}
$$

where

$$
h_{x, y}=h(-, x) y-h(-, y) x
$$

and

$$
h_{0}(x, y)=h(x, y)-\langle x \mid y\rangle=\frac{1}{2}(h(x, y)-h(y, x)) .
$$

Note that

$$
h_{x, y} \in \mathfrak{u}(V, h)=\left\{f \in \operatorname{End}_{K}(V): h(f(x), y)+h(x, f(y))=0 \text { for any } x, y \in V\right\} .
$$

Since $\overline{h_{0}(x, y)}=-h_{0}(x, y)$, it follows that $\mathfrak{d} \subseteq \mathfrak{u}(V, h)$.

In the split case: $K=F \times F=F e_{1} \oplus F e_{2}$, for orthogonal idempotents $e_{1}$ and $e_{2}$ $\left(\bar{e}_{1}=e_{2}\right)$, let $W=e_{1} V$ and $\tilde{W}=e_{2} V$. Then $h(W, W)=0=h(\tilde{W}, \tilde{W})$ and for any $a \in W$ and $u \in \tilde{W}, h(a, u) \in F e_{1}$. Hence there is a bilinear nondegenerate form (|): $W \times \tilde{W} \rightarrow F$, such that $h(a, u)=(a \mid u) e_{1}$ for any $a \in W$ and $u \in \tilde{W}$. This bilinear form determines $h$ and allows us to identify $\tilde{W}$ with the dual $W^{*}$. Therefore we may assume that $V=W \times W^{*}$, with the natural structure of module over $K=F \times F$, and with $h((a, \alpha),(b, \beta))=(\beta(a), \alpha(b))$ for any $a, b \in W$ and $\alpha, \beta \in W^{*}$. Moreover, in this case $\mathfrak{u}(V, h)$ is isomorphic to $\mathfrak{g l}(W)$ by means of the isomorphism that takes any $f \in \operatorname{End}_{F}(W)=\mathfrak{g l}(W)$ to the endomorphism of $V=W \times W^{*}$ given by $(a, \alpha) \mapsto$ $(f(a),-\alpha \circ f)$. Through this isomorphism, $h_{(a, 0),(0, \alpha)}$ corresponds to the endomorphism of $W$ given by $c \mapsto-\alpha(c) a$, and hence $d_{(a, 0),(0, \alpha)}$ corresponds to $c \mapsto-\alpha(c) a+\frac{1}{2} \alpha(a) c$. If the dimension of $W$ is not 2 , this shows that $\mathfrak{d}=\mathfrak{u}(V, h) \cong \mathfrak{g l}(W)$, while if the dimension is $2, \mathfrak{d}=\mathfrak{s u}(V, h) \cong \mathfrak{s l}(W)$.

By scalar extension, we have that $\mathfrak{d}=\mathfrak{u}(V, h)$ if $\operatorname{dim}_{K} V \neq 2\left(\operatorname{dim}_{F} V \neq 4\right)$ and $\mathfrak{d}=\mathfrak{s u}(V, h)$ if $\operatorname{dim}_{K} V=2$.

Finally, assume that $R$ is a quaternion algebra $Q$. Again $d_{x, y}=h_{x, y}+h_{0}(x, y) 1_{V}$, but now $h_{x, y}$ is $Q$-linear, while $h_{0}(x, y) 1_{V}$ is not in general, since the center of $Q$ is $F$. It is easily checked here that $\left[h_{x, y}, h_{u, v}\right]=h_{h_{x, y}(u), v}+h_{u, h_{x, y}(v)}$ for any $x, y, u, v \in V$, and thus $h_{V, V}=\operatorname{span}\left\{h_{x, y}: x, y \in V\right\}$ is a Lie algebra contained in

$$
\mathfrak{s p}(V, h)=\left\{f \in \operatorname{End}_{Q}(V): h(f(x), y)+h(x, f(y))=0 \text { for any } x, y \in V\right\},
$$

and $\mathfrak{d}=d_{V, V}$ is contained in $\mathfrak{s p}(V, h) \oplus Q_{0} 1_{V}$, where $Q_{0}=[Q, Q]$ is the set of skew symmetric elements in $Q$ relative to its involution, which form a three dimensional simple Lie algebra.

Again, consider the split case: $Q=\operatorname{End}_{F}(U)$ for a two dimensional vector space $U$ endowed with a nonzero skew symmetric bilinear $\operatorname{map} \varphi$ which induces the involution in $Q$. Standard arguments of complete reducibility as a module over $Q$ show that $V=U \otimes_{F} W$ for some vector space $W$ over $F$. For any $q \in Q_{0}=\mathfrak{s l}(U)=\mathfrak{s p}(U, \varphi)$ 
and for any $x, y \in V$,

$$
\begin{aligned}
\langle q x \mid y\rangle & =\frac{1}{2}(h(q x, y)+h(y, q x))=\frac{1}{2}(q h(x, y)+\overline{q h(x, y)}) \\
& =\frac{1}{2}(h(x, y) q+\overline{h(x, y) q})=-\langle x \mid q y\rangle,
\end{aligned}
$$

so $Q_{0}$ embeds into the orthogonal Lie algebra $o(V,\langle\mid\rangle)$ and, therefore, $\langle\mid\rangle$ is invariant under the action of $\mathfrak{s l}(U)=\mathfrak{s p}(U, \varphi)$. But, up to scalars, $\varphi$ is the unique bilinear form on $U$ which is $\mathfrak{s p}(U, \varphi)$-invariant, so $\langle a \otimes u \mid b \otimes v\rangle=\frac{1}{2} \varphi(a, b) \psi(u, v)$, for any $a, b \in U$, $u, v \in W$, for a skew-symmetric nondegenerate bilinear form $\psi: W \times W \rightarrow F$.

Since the hermitian form $h$ is completely determined by $\langle\mid\rangle$, it turns out that $h$ : $V \times V \rightarrow Q=\operatorname{End}_{F}(U)$ is given by $h(a \otimes u, b \otimes v)=\psi(u, v) \varphi(-, b) a$, for any $a, b \in$ $U$ and $u, v \in W$. Note that $h$ thus defined is hermitian and

$$
\frac{1}{2}(h(a \otimes u, b \otimes v)+h(b \otimes v, a \otimes u))=\frac{1}{2} \psi(u, v)(\varphi(-, b) a-\varphi(-, a) b) .
$$

But $\varphi(a, b) c+\varphi(b, c) a+\varphi(c, a) b=0$ for any $a, b, c \in U$, so

$$
\frac{1}{2}(h(a \otimes u, b \otimes v)+h(b \otimes v, a \otimes u))=\frac{1}{2} \psi(u, v) \varphi(a, b) 1_{V} .
$$

Hence, for any $a, b \in U$ and $u, v \in W$ :

$$
\begin{aligned}
h_{0}(a \otimes u, b \otimes v) & =\frac{1}{2}(h(a \otimes u, b \otimes v)-h(b \otimes v, a \otimes u)) \quad(\text { see (3.6)) } \\
& =\frac{1}{2} \psi(u, v)(\varphi(-, b) a+\varphi(-, a) b)=\frac{1}{2} \psi(u, v) \varphi_{a, b},
\end{aligned}
$$

and thus, for any $a, b, c \in U$ and $u, v, w \in W$ :

$$
\begin{aligned}
h_{a \otimes u, b \otimes v}(c \otimes w) & =\psi(w, u) \varphi(b, a) c \otimes v-\psi(w, v) \varphi(a, b) c \otimes u \\
& =-\varphi(a, b) c \otimes(\psi(w, u) v+\psi(w, v) u)=-\varphi(a, b) c \otimes \psi_{u, v}(w) .
\end{aligned}
$$

Therefore, $h_{V, V}=\mathfrak{s p}(V, h):=\left\{f \in \operatorname{End}_{Q}(V): h(f(x), y)+h(x, f(y))=0\right.$ for any $x, y \in V\} \cong \mathfrak{s p}(W, \psi)$ (which acts on $V=U \otimes_{F} W$ in a natural way: on the second factor). Moreover, from (3.4),

$$
d_{a \otimes u, b \otimes v}=h_{a \otimes u, b \otimes v}+h_{0}(a \otimes u, b \otimes v) 1_{V}=\frac{1}{2} \varphi_{a, b} \otimes \psi(u, v) 1_{W}-\varphi(a, b) 1_{U} \otimes \psi_{u, v},
$$

so $\mathfrak{d}=d_{V, V}=\mathfrak{s p}(U, \varphi) \oplus \mathfrak{s p}(W, \psi)=\mathfrak{s l}(U) \oplus \mathfrak{s p}(W, \psi)$.

For general $Q$, again extending scalars we arrive at $h_{V, V}=\mathfrak{s p}(V, h)$ (which is a simple Lie algebra of type $C$ ) and $\mathfrak{d}$ is the direct sum of the three dimensional simple Lie algebra $Q_{0}$ and of the simple Lie algebra $\mathfrak{s p}(V, h)$.

Summarizing the above discussion:

Proposition 3.1. Let $R$ be a unital separable associative algebra of degree $\leq 2$ over a field $F$ of characteristic $\neq 2$, and let $V$ be a left module over $R$ endowed with a nondegenerate hermitian form $h: V \times V \rightarrow R$. Endow $V$ with the structure of a simple $(-1,-1)$-BFKTS of hermitian type (with associated symmetric bilinear form given by 
$\langle x \mid y\rangle=\frac{1}{2}(h(x, y)+h(y, x))$ for any $\left.x, y \in V\right)$ and let $\mathfrak{d}=d_{V, V}$ be the associated Lie algebra of inner derivations. Then:

(i) If $R=F$, then $\mathfrak{d}=\mathfrak{o}(V,\langle\mid\rangle)$.

(ii) If $R=K$ is a quadratic étale algebra, then $\mathfrak{d}=\mathfrak{u}(V, h)$ unless $\operatorname{dim}_{F}(V)=4$. In this latter case, $\mathfrak{d}=\mathfrak{s u}(V, h)$.

(iii) If $R$ is a quaternion algebra $Q$, then $\mathfrak{d} \cong Q_{0} \oplus \mathfrak{s p}(V, h)$, where $\mathfrak{s p}(V, h)$ acts naturally on $V$, and the simple three dimensional Lie algebra $Q_{0}$ acts by left multiplication on the $Q$ module $V$.

3.2. $\mathbf{D}_{\mu}$-type. Let $V$ be a four dimensional vector space, endowed with a nondegenerate symmetric bilinear form $\langle\mid\rangle$. Let $\Phi$ be a nonzero skew symmetric multilinear form: $\Phi: V \times V \times V \times V \rightarrow F$. Define a skew symmetric triple product $[x y z]$ on $V$ by means of:

$$
\Phi(x, y, z, t)=\langle[x y z] \mid t\rangle,
$$

for any $x, y, z, t \in V$. The proof of the next result is left to the reader.

LEMMA 3.2. With the hypotheses above, there exists a nonzero scalar $\mu \in F$ such that

$$
\left\langle\left[a_{1} a_{2} a_{3}\right] \mid\left[b_{1} b_{2} b_{3}\right]\right\rangle=\mu \operatorname{det}\left(\left\langle a_{i} \mid b_{j}\right\rangle\right),
$$

for any $a_{i}, b_{i} \in V(i=1,2,3)$.

Now, for any such $V$ and $\Phi$, and for any $x, y \in V$, consider the endomorphism $d_{x, y} \in \operatorname{End}_{F}(V)$ defined by

$$
d_{x, y} z=[x y z]+\langle z \mid x\rangle y-\langle z \mid y\rangle x .
$$

As shown in $[22, \S 5]$, conditions $(2.3 \mathrm{a}-\mathrm{b})$ are satisfied, so if the triple product $x y z$ on $V$ is defined by means of

$$
x y z=[x y z]+\langle z \mid x\rangle y-\langle z \mid y\rangle x+\langle x \mid y\rangle z .
$$

for any $x, y, z \in V$, then $V$ becomes a $(-1,-1)$-BFKTS, which will be said to be of $\mathrm{D}_{\mu}$-type.

Assume for a while that the scalar $\mu$ in (3.8) is a square, $\mu=v^{2}, 0 \neq v \in F$, and that $\langle\mid\rangle$ represents 1 . Then, by [4, Theorem 2], $V$ is endowed with a binary multiplication that makes it a quaternion algebra $Q$ over $F$, with involution $x \mapsto \bar{x}$ such that $x \bar{x}=\langle x \mid x\rangle$ for any $x \in V$, satisfying

$$
v^{-1}[x y z]=x \bar{y} z-\langle x \mid y\rangle z+\langle z \mid x\rangle y-\langle z \mid y\rangle x
$$

for any $x, y, z \in V$. Therefore, for any $x, y, z \in V,(3.9)$ shows that:

$$
\begin{aligned}
d_{x, y}(z) & =v x \bar{y} z+(1+v)(\langle z \mid x\rangle y-\langle z \mid y\rangle x)-v\langle x \mid y\rangle z \\
& =v x \bar{y} z+\frac{1+v}{2}((x \bar{z}+z \bar{x}) y-x(\bar{y} z+\bar{z} y))-\frac{v}{2}(x \bar{y}+y \bar{x}) z \\
& =\left(v x \bar{y}-\frac{1+v}{2} x \bar{y}-\frac{v}{2}(x \bar{y}+y \bar{x})\right) z+\frac{1+v}{2} z \bar{x} y \\
& =\left(-\frac{1}{2} x \bar{y}-\frac{v}{2} y \bar{x}\right) z+\frac{1+v}{2} z \bar{x} y=\frac{v-1}{4}(x \bar{y}-y \bar{x}) z+\frac{1+v}{4} z(\bar{x} y-\bar{y} x),
\end{aligned}
$$


because $\bar{x} y+\bar{y} x=x \bar{y}+y \bar{x}=2\langle x \mid y\rangle \in F$, so $\bar{x} y-\bar{y} x=2 \bar{x} y-(x \bar{y}+y \bar{x})$. Hence, for any $x, y \in V, d_{x, y}=L_{p}-R_{q}$, with $p=\frac{v-1}{4}(x \bar{y}-y \bar{x}), q=-\frac{v+1}{4}(\bar{x} y-\bar{y} x) \in Q_{0}$, where $L$ and $R$ denote left and right multiplications in $V=Q$. Therefore, if $\mu=1$ $(v= \pm 1), \mathfrak{d}=d_{V, V}$ is isomorphic to the three dimensional simple Lie algebra $Q_{0}$. However, if $\mu \neq 0,1(v \neq 0, \pm 1)$, then $\mathfrak{d}=L_{Q_{0}} \oplus R_{Q_{0}}$, a direct sum of two copies of the three dimensional Lie algebra $Q_{0}$, which coincides with the orthogonal Lie algebra $\mathfrak{o}(V,\langle\mid\rangle)$. Moreover, in this latter case, [2, Lemma 3.1 and its proof ], $\mathfrak{g}(V)$ is a form of the simple Lie superalgebra $\Gamma\left(-\frac{1}{2}, \frac{1-v}{4}, \frac{1+v}{4}\right)$ (notation as in [26, pp. 16-17]). That is, it is a form of $D\left(2,1 ; \frac{v-1}{2}\right)$ (see also [19]).

Simply by extending scalars, we obtain:

Proposition 3.3. Let $V$ be a four dimensional vector space over a field $F$ of characteristic $\neq 2$ with a nondegenerate symmetric bilinear form $\langle\mid\rangle$. Let $\Phi$ be a nonzero skew symmetric 4-linear form and let the triple product $[x y z]$ be defined by means of $\langle[x y z] \mid t\rangle=\Phi(x, y, z, t)$ for any $x, y, z, t \in V$. Let $0 \neq \mu \in F$ be given by (3.8). Endow $V$ with the structure of a simple $(-1,-1)$-BFKTS by means of $(3.10)$ and let $\mathfrak{d}=d_{V, V}$ be the corresponding Lie algebra of inner derivations. Then:

(i) If $\mu=1$, then $\mathfrak{d}$ is a three dimensional simple ideal of the orthogonal Lie algebra $\mathfrak{o}(V,\langle\mid\rangle)$.

(ii) If $\mu \neq 0,1$, then $\mathfrak{d}$ coincides with the orthogonal Lie algebra $\mathfrak{o}(V,\langle\mid\rangle)$.

There is some overlapping in the types considered up to now.

To begin with, let $V$ be any four dimensional simple $(-1,-1)$-BFKTS and let $[x y z]$ be defined by $[x y z]=x y z-\langle z \mid x\rangle y+\langle z \mid y\rangle x-\langle x \mid y\rangle z$, for any $x, y, z \in V$. Because of $(2.5 \mathrm{a}),[x y z]$ is skew symmetric on its arguments. In case $[x y z]$ is identically zero, we are in presence of a system of orthogonal type. Otherwise, this is a system of D-type. This means that the systems of hermitian type with $R=K$ or $Q$ and with $\operatorname{dim}_{F} V=4$ are systems of D-type. Let us check which $\mu$ 's are involved in these cases. To do so, it is enough to consider the split cases.

Assume $K=F \times F$ and $V=W \times W^{*}$ with $h((a, \alpha),(b, \beta))=(\beta(a), \alpha(b))$ for any $a, b \in W$ and $\alpha, \beta \in W$ and with $\operatorname{dim}_{F} W=2$. Take $a, b \in W$ and $\alpha, \beta \in W^{*}$ with $\alpha(a)=1=\beta(b), \alpha(b)=0=\beta(a)$. Then with $\left(a_{1}, \alpha_{1}\right)=(a, 0),\left(a_{2}, \alpha_{2}\right)=(0, \alpha)$ and $\left(a_{3}, \alpha_{3}\right)=(b, \beta)$,

$$
\operatorname{det}\left(\left\langle\left(a_{i}, \alpha_{i}\right) \mid\left(a_{j}, \alpha_{j}\right)\right\rangle\right)=\left|\begin{array}{ccc}
0 & \frac{1}{2} & 0 \\
\frac{1}{2} & 0 & 0 \\
0 & 0 & 1
\end{array}\right|=-\frac{1}{4},
$$

while $[(a, 0)(0, \alpha)(b, \beta)]=\frac{1}{2}(b, \beta)$ and

$$
\langle[(a, 0)(0, \alpha)(b, \beta)] \mid[(a, 0)(0, \alpha)(b, \beta)]\rangle=\frac{1}{4}\langle(b,-\beta) \mid(b,-\beta)\rangle=-\frac{1}{4} .
$$

Hence, $\mu=1$ in this case. (This can also be deduced directly from the size of the Lie algebras do.)

Assume now that $R=Q$ is a quaternion algebra and $\operatorname{dim}_{F} V=4$, then $V$ is a free $Q$-module of rank 1 and hence we may assume that $V=Q$ and that $h(x, y)=\alpha x \bar{y}$ for any $x, y \in Q$, where $0 \neq \alpha=h(1,1) \in F$. Then for any $x_{1}, x_{2}, x_{3} \in Q$,

$$
\left[x_{1} x_{2} x_{3}\right]=h_{0}\left(x_{3}, x_{1}\right) x_{2}-h_{0}\left(x_{3}, x_{2}\right) x_{1}+h_{0}\left(x_{1}, x_{2}\right) x_{3}
$$


where $h_{0}(x, y)=\frac{1}{2}(h(x, y)-h(y, x))=\alpha(x \bar{y}-y \bar{x}) \in Q_{0}$. By skew symmetry of $h_{0}$,

$$
\left[x_{1} x_{2} x_{3}\right]=\frac{1}{2} \sum_{\sigma} h_{0}\left(x_{\sigma(1)}, x_{\sigma(2)}\right) x_{\sigma(3)}=\frac{\alpha}{2}(-1)^{\sigma} x_{\sigma(1)} \bar{x}_{\sigma(2)} x_{\sigma(3)}
$$

where the sum is over all the permutations of $1,2,3$. Take $x_{1}=1, x_{2}$, and $x_{3}$ mutually orthogonal to get $\langle 1 \mid 1\rangle=h(1,1)=\alpha, \operatorname{det}\left(\left\langle x_{i} \mid x_{j}\right\rangle\right)=\alpha\left\langle x_{2} \mid x_{2}\right\rangle\left\langle x_{3} \mid x_{3}\right\rangle$, while $\left[x_{1} x_{2} x_{3}\right]=-3 \alpha x_{2} x_{3}$ since $x_{2} x_{3}=-x_{3} x_{2}, \bar{x}_{i}=-x_{i}$ for $i=2,3$, and $\overline{1}=1$. Thus, $\left\langle\left[x_{1} x_{2} x_{3}\right] \mid\left[x_{1} x_{2} x_{3}\right]\right\rangle=9 \alpha^{3}\left(x_{2} x_{3}\right) \overline{\left(x_{2} x_{3}\right)}=9 \alpha\left\langle x_{2} \mid x_{2}\right\rangle\left\langle x_{3} \mid x_{3}\right\rangle$, and $\mu=9$ in this case.

A final overlap occurs if $V$ is of hermitian type with $R=K$ quadratic and with $\operatorname{dim}_{F} V=2$. As above, $\left[x_{1} x_{2} x_{3}\right]=\frac{1}{2} \sum_{\sigma} h_{0}\left(x_{\sigma(1)}, x_{\sigma(2)}\right) x_{\sigma(3)}$ for any $x_{1}, x_{2}, x_{3} \in V$. By skew symmetry and dimension, this is zero, and therefore we are in the situation of $R=F$. We summarize the above arguments in the following remark, whose last part follows from the structure of the Lie algebras of inner derivations.

REMARK 3.4.

- The simple $(-1,-1)$-BFKTS $V$ of unitarian type and $\operatorname{dim}_{F} V=2$ are also of orthogonal type.

- The simple $(-1,-1)$-BFKTS $V$ of unitarian type and $\operatorname{dim}_{F} V=4$ are of $\mathrm{D}_{1}$ type.

- The simple $(-1,-1)$-BFKTS $V$ of symplectic type and $\operatorname{dim}_{F} V=4$ are of D9-type.

- There are no more overlaps among different types.

3.3. G-type. Let $C$ be an eight-dimensional Cayley-Dickson (or octonion) algebra over $F$ with norm $n$ and trace $t$. Let $C_{0}$ be the set of trace zero elements. For any $x, y \in C$, the linear map

$$
D_{x, y}=\left[L_{x}, L_{y}\right]+\left[L_{x}, R_{y}\right]+\left[R_{x}, R_{y}\right]
$$

(where $L_{x}$ and $R_{x}$ denote the left and right multiplication by $x$ ) is known to be a derivation of $C$ [25, Ch. III.8], and hence it leaves $C_{0}$ invariant. Consider then, for any $0 \neq \alpha \in F$, the nondegenerate symmetric bilinear form and the triple product on $V=C_{0}$ given by $\langle x \mid y\rangle=-2 \alpha t(x y)$ and $x y z=\alpha\left(D_{x, y}(z)-2 t(x y) z\right)$, for any $x, y, z \in$ $V$. Since $D_{x, y}$ is a derivation and

$$
\begin{aligned}
D_{x, y}(y) & =x y^{2}-y(x y)+x y^{2}-(x y) y+y^{2} x-(y x) y=4 y^{2} x-2(x y+y x) y \\
& =-4 n(y) x-2 t(x y) y=-\langle y \mid y\rangle x+\langle x \mid y\rangle y,
\end{aligned}
$$

where we have used that $x^{2}=-n(x) 1=-\frac{1}{2} t\left(x^{2}\right) 1$ for any $x \in V=C_{0}$; it follows from (2.3) that $V$ is a $(-1,-1)$-BFKTS (see also [19]), which will be said to be of G-type. It is clear here that the Lie algebra $\mathfrak{d}$ is the span of the $D_{x, y}$ 's, which is precisely the Lie algebra of derivations of the Cayley-Dickson algebra $C$ in case the characteristic is $\neq 3$ [25, Ch. III.8], a simple Lie algebra of type $G_{2}$. (If the characteristic is 3 , then this is a seven dimensional simple Lie algebra which is a form of $\mathfrak{p s t}(7)$ [1].)

3.4. F-type. Let $X$ be a 3 -fold vector cross product on a vector space $V$ of dimension 8 , endowed with a nondegenerate symmetric bilinear form $\langle\mid\rangle$. That is, $X$ is a trilinear map $X: V \times V \times V \rightarrow V,(a, b, c) \mapsto X(a, b, c)$, satisfying (see [4], [23, 
Ch. 8] and the references therein):

$$
\begin{gathered}
\left\langle X\left(a_{1}, a_{2}, a_{3}\right) \mid a_{i}\right\rangle=0 \text { for any } i=1,2,3, \\
\left\langle X\left(a_{1}, a_{2}, a_{3}\right) \mid X\left(a_{1}, a_{2}, a_{3}\right)\right\rangle=\operatorname{det}\left(\left\langle a_{i} \mid a_{j}\right\rangle\right),
\end{gathered}
$$

for any $a_{1}, a_{2}, a_{3} \in V$.

It is known that (3.12) implies the skew symmetry of $X$. Moreover, $X$ satisfies:

$$
\begin{aligned}
& \left\langle X\left(a_{1}, a_{2}, a_{3}\right) \mid X\left(b_{1}, b_{2}, b_{3}\right)\right\rangle \\
& =\operatorname{det}\left(\left\langle a_{i} \mid b_{j}\right\rangle\right)+\epsilon \sum_{\sigma \text { even }} \sum_{\tau \text { even }}\left\langle a_{\sigma(1)} \mid b_{\tau(1)}\right\rangle \Phi\left(a_{\sigma(2)}, a_{\sigma(3)}, b_{\tau(2)}, b_{\tau(3)}\right)
\end{aligned}
$$

for any $a_{i}, b_{i} \in V(i=1,2,3)$, where $\Phi(a, b, c, d)=\langle a \mid X(b, c, d)\rangle$ for any $a, b, c, d \in$ $V$, and $\epsilon= \pm 1$. In case $\epsilon=1$ (resp. -1 ), $X$ is said to be of type I (resp. II). Also, if $\operatorname{dim}_{F} V=8$ and $X$ is of type I, then $-X$ is of type II, and conversely.

Assume now that the characteristic of the ground field $F$ is $\neq 2,3$. Given a 3 -fold vector cross product $X$ of type I, define $d_{x, y} \in \operatorname{End}_{F}(V), x, y \in V$, by means of:

$$
d_{x, y} z=\frac{1}{3} X(x, y, z)+\langle z \mid x\rangle y-\langle z \mid y\rangle x
$$

As shown in [22, $\S 5]$, condition (2.3b) is satisfied, so if the triple product $x y z$ on $V$ is defined by means of

$$
x y z=\frac{1}{3} X(x, y, z)+\langle z \mid x\rangle y-\langle z \mid y\rangle x+\langle x \mid y\rangle z
$$

for any $x, y, z \in V$, then $V$ becomes a $(-1,-1)$-BFKTS, which will be said to be of F-type.

Since $d_{x, y}$ is a derivation of the triple system and it is skew symmetric relative to $\langle\mid\rangle$, it follows that $d_{x, y}$ is a derivation of the 3 -fold vector cross product $X$. According to [4, Theorem 12], if $e$ is an element of $V$ with $\langle e \mid e\rangle \neq 0, W=\{v \in V:\langle e \mid x\rangle=0\}$, and $q$ is the nondegenerate quadratic form on $V$ defined by $q(v)=-\langle e \mid e\rangle^{-1}\langle v \mid v\rangle$, then the Lie algebra of derivations of $X$ is isomorphic to the orthogonal Lie algebra $\mathfrak{o}(W, q)$. Actually, $V$ has the structure of an eight dimensional Cayley-Dickson algebra $C$ with unit $1=e$, so that there is an scalar $0 \neq \alpha \in F$ such that $X(a, b, c)=\alpha((a \bar{b}) c+$ $(a \mid c) b-(b \mid c) a-(a \mid b) c)$ and $\langle a \mid b\rangle=\alpha(a \mid b)$, for any $a, b, c \in V=C$. Here $x \mapsto \bar{x}$ denotes the involution and $(a \mid a)=a \bar{a}$ is the norm of $C$. Note that $\alpha=\langle e \mid e\rangle$. Hence for any $x, y \in V, d_{x, y}$ is a derivation of the $3 C$-product given by $(a \bar{b}) c$ (see [4]). But for any $x, y, z \in V=C, \frac{3}{\alpha} d_{x, y}(z)=(x \bar{y}) z+4(z \mid x) y-4(z \mid x) x-(x \mid y) z$, in particular, for a traceless $x(\bar{x}=-x), \frac{3}{\alpha} d_{e, x}(y)=-x y+2 x(y+\bar{y})-2(x \bar{y}-y x)=x y+2 y x=$ $(L+2 R)_{x}(y)$, that is, $d_{e, x}=(L+2 R)_{x}$, where $L$ and $R$ denote the left and right multiplications in $C$. But these operators generate the Lie algebra of derivations of the triple product given by $(a \bar{b}) c[7,4]$ (see also [5]), so we conclude that $\mathfrak{d}$ is isomorphic to $\mathfrak{o}(W, q)$.

Note that in [19] it is already proved that, after scalar extension, $\mathfrak{d}$ is isomorphic to $\mathfrak{o}(7)$, by an explicit computation. 
4. Classification. Given a $(-1,-1)$-BFKTS $V$ over a field of characteristic $\neq 2$, in Section 2 a simple Lie superalgebra $\mathfrak{g}=\mathfrak{g}(V)$ has been defined that contains a copy $\mathfrak{s}=\mathfrak{s}(V)$ of $\mathfrak{s l}_{2}(F)$, which is an ideal of $\mathfrak{g}_{0}$ that is complemented by the ideal $\mathfrak{d}=\mathfrak{d}(V)=d_{V, V}$. In this situation $\mathfrak{d}=\left\{d \in \mathfrak{g}_{0}:[d, \mathfrak{s}]=0\right\}$ is completely determined by $\mathfrak{g}$ and $\mathfrak{s}$. Moreover, as a module for $\mathfrak{g}_{\overline{0}}, \mathfrak{g}_{\overline{1}}$ is the tensor product of the two dimensional irreducible module for $\mathfrak{s}$ and the module $V$ for $\mathfrak{d}$.

Consider a ground field $F$ of characteristic $\neq 2$ and the pairs $(\mathfrak{g}, \mathfrak{s})$, where $\mathfrak{g}$ is a Lie superalgebra over $F$ and $\mathfrak{s}$ is a complemented ideal of $\mathfrak{g}_{0}$ isomorphic to $\mathfrak{s l}_{2}(F)$. Two such pairs $\left(\mathfrak{g}^{1}, \mathfrak{s}^{1}\right),\left(\mathfrak{g}^{2}, \mathfrak{s}^{2}\right)$ are said to be isomorphic if there is an isomorphism of Lie superalgebras $\phi: \mathfrak{g}^{1} \rightarrow \mathfrak{g}^{2}$ such that $\phi\left(\mathfrak{s}^{1}\right)=\mathfrak{s}^{2}$.

Given a Lie superalgebra $\mathfrak{g}=\mathfrak{g}_{\overline{0}} \oplus \mathfrak{g}_{\overline{1}}$ and a nonzero scalar $\alpha$, the new Lie superalgebra defined over $\mathfrak{g}$ with the new product $[,]_{\alpha}$ given, for homogeneous elements, by

$$
\begin{cases}{[x, y]_{\alpha}=\alpha[x, y]} & \text { if both } x \text { and } y \text { are odd } \\ {[x, y]_{\alpha}=[x, y]} & \text { otherwise }\end{cases}
$$

will be denoted by $\mathfrak{g}_{\alpha}$. Also, given a $(-1,-1)$-BFKTS $V$, we will denote by $V_{\alpha}$ the new $(-1,-1)$-BFKTS defined on $V$ but with the new product given by $(x y z)_{\alpha}=\alpha x y z$, and new symmetric bilinear form given by $\langle x \mid y\rangle_{\alpha}=\alpha\langle x \mid y\rangle$, for any $x, y, z \in V$. From the definitions, it is clear that $\mathfrak{g}\left(V_{\alpha}\right)=\mathfrak{g}(V)_{\alpha}$. Two $(-1,-1)$-BFKTS $V^{1}$ and $V^{2}$ will be said to be equivalent in case there is a nonzero scalar $\alpha$ such that $V^{1}$ and $V_{\alpha}^{2}$ are isomorphic.

THEOREM 4.1. Let $V^{1}$ and $V^{2}$ be two $(-1,-1)$-BFKTS's. Then $V^{1}$ is equivalent to $V^{2}$ if and only if $\left(\mathfrak{g}\left(V^{1}\right), \mathfrak{s}\left(V^{1}\right)\right)$ is isomorphic to $\left(\mathfrak{g}\left(V^{2}\right), \mathfrak{s}\left(V^{2}\right)\right)$.

Proof. Let $\mathfrak{g}^{i}=\mathfrak{g}\left(V^{i}\right)$ and $\mathfrak{d}^{i}=\mathfrak{d}\left(V^{i}\right)=d_{V^{i}, V^{i}}$ for $i=1$, 2. Also, $\mathfrak{s}\left(V^{1}\right)=\mathfrak{s}\left(V^{2}\right)=$ $\mathfrak{s p}(U, \varphi)$ as in Section 2. Thus $\mathfrak{g}_{\overline{0}}^{i_{\overline{0}}}=\mathfrak{s p}(U, \varphi) \oplus \mathfrak{d}^{i}$ and $\mathfrak{g}_{\overline{1}}^{i_{\overline{1}}}=U \otimes_{F} V^{i}$, for $i=1$, 2. Let $\Phi: \mathfrak{g}^{1} \rightarrow \mathfrak{g}^{2}$ be an isomorphism such that it restricts to an automorphism of $\mathfrak{s p}(U, \varphi)$. But any automorphism $\xi$ of $\mathfrak{s p}(U, \varphi)$ can be extended as in [6, proof of Lemma 2.1] to an isomorphism from $\mathfrak{g}^{2}$ onto $\mathfrak{g}_{\alpha}^{2}$ for some nonzero scalar $\alpha$ and, therefore, we may (and will) assume that $\Phi$ is the identity on $\mathfrak{s p}(U, \varphi)$. Since $\mathfrak{d}^{i}$ is the centralizer of $\mathfrak{s p}(U, \varphi)$ in $\mathfrak{g}_{0}^{i}, i=1,2, \Phi$ restricts to an isomorphism $\Psi: \mathfrak{d}^{1} \rightarrow \mathfrak{d}^{2}$. Also, $\Phi$ restricts then to an isomorphism of $\mathfrak{s p}(U, \varphi)$-modules $\Phi_{\overline{1}}: U \otimes_{F} V^{1} \rightarrow U \otimes_{F} V^{2}$. Since $U$ is absolutely irreducible as a module for $\mathfrak{s p}(U, \varphi)$, there is an isomorphism of vector spaces $\psi: V^{1} \rightarrow V^{2}$ such that $\Phi(a \otimes x)=a \otimes \psi(x)$ for any $a \in U$ and $x \in V^{1}$.

Now, for any $x, y, z \in V^{1}$ and any $a \in U$, we have $a \otimes \psi\left(d_{x, y}(z)\right)=$ $\Phi\left(\left[d_{x, y}, a \otimes z\right]\right)=\left[\Psi\left(d_{x, y}\right), a \otimes \psi(z)\right]=a \otimes \Psi\left(d_{x, y}\right)(\psi(z))$, so

$$
\psi\left(d_{x, y}(z)\right)=\Psi\left(d_{x, y}\right)(\psi(z))
$$

for any $x, y, z \in V^{1}$. Also, for any $a, b \in U$ and $x, y \in V^{1}$ we have $\Phi([a \otimes x, b \otimes y])=$ $[a \otimes \psi(x), b \otimes \psi(y)]=\langle\psi(x) \mid \psi(y)\rangle \varphi_{a, b}+\varphi(a, b) d_{\psi(x), \psi(y)}$, but also $\Phi([a \otimes x, b \otimes y])=$ $\Phi\left(\langle x \mid y\rangle \varphi_{a, b}+\varphi(a, b) d_{x, y}\right)=\langle x \mid y\rangle \varphi_{a, b}+\varphi(a, b) \Psi\left(d_{x, y}\right)$. So

$$
\left\{\begin{array}{l}
\Psi\left(d_{x, y}\right)=d_{\psi(x), \psi(y)}, \\
\langle\psi(x) \mid \psi(y)\rangle=\langle x \mid y\rangle,
\end{array}\right.
$$

for any $x, y \in V^{1}$, which, together with (4.1), shows that $\psi$ is an isomorphism between the triple systems $V^{1}$ and $V^{2}$. 
For the converse, if $V^{1}$ and $V^{2}$ are equivalent, there is a $0 \neq \alpha \in F$ such that $V^{1}$ and $V_{\alpha}^{2}$ are isomorphic. From here it is easy to deduce that the pairs $\left(\mathfrak{g}\left(V^{1}\right), \mathfrak{s}\left(V^{1}\right)\right)$ and $\left(\mathfrak{g}\left(V_{\alpha}^{2}\right), \mathfrak{s}\left(V^{2}\right)\right)$ are isomorphic. But $\mathfrak{g}\left(V_{\alpha}^{2}\right)$ is isomorphic to $\mathfrak{g}\left(V^{2}\right)$ by means of an isomorphism taking $\mathfrak{s}\left(V^{2}\right)$ into itself (see [6, proof of Lemma 2.1]).

In order to classify the simple $(-1,-1)$-BFKTS of finite dimension over a field of characteristic zero, we will first assume that the ground field $F$ is algebraically closed. Following Theorems 2.1, 2.2 and 4.1, we will determine, up to isomorphism, the pairs $(\mathfrak{g}, \mathfrak{s})$, where $\mathfrak{g}$ is a simple finite dimensional Lie superalgebra and $\mathfrak{s}$ is an ideal of $\mathfrak{g}_{\overline{0}}$ isomorphic to $\mathfrak{s l}(2)$ :

THEOREM 4.2. Let $F$ be an algebraically closed field of characteristic zero. The following list exhausts, up to isomorphism, the pairs $(\mathfrak{g}, \mathfrak{s})$, where $\mathfrak{g}$ is a simple finite dimensional Lie superalgebra over $F\left(\mathfrak{g}_{\overline{1}} \neq 0\right)$ and $\mathfrak{s}$ is a three dimensional simple ideal of $\mathfrak{g}_{\overline{0}}$.

(i) $\mathfrak{g}=\mathfrak{s l}(m, 2), m \geq 3$, and $\mathfrak{s}$ is the (unique) ideal of $\mathfrak{g}_{0}$ isomorphic to $\mathfrak{s l}(2)$.

(ii) $\mathfrak{g}=\mathfrak{p s l}(2,2)$ and $\mathfrak{s}$ is any of the two simple ideals of $\mathfrak{g}_{\overline{0}}$. $\mathfrak{s p}(2)$.

(iii) $\mathfrak{g}=\mathfrak{o s p}(m, 2), m \geq 1, m \neq 4$, so that $\mathfrak{g}_{\overline{0}}=\mathfrak{o}(m) \oplus \mathfrak{s p}(2)$, and $\mathfrak{s}$ is the copy of

(iv) $\mathfrak{g}=\mathfrak{o s p}(4,2 r), r \geq 2$, so that $\mathfrak{g}=\mathfrak{o}(4) \oplus \mathfrak{s p}(2 r)$ and $\mathfrak{s}$ is any of the two simple simple ideals of $\mathfrak{o}(4) \cong \mathfrak{s l}(2) \oplus \mathfrak{s l}(2)$.

(v) $\mathfrak{g}=D(2,1 ; \alpha), \quad \alpha \neq 0,-1$, so that $\mathfrak{g}_{0}=\mathfrak{s p}(U, \varphi) \oplus \mathfrak{s p}(U, \varphi) \oplus \mathfrak{s p}(U, \varphi), \quad U$ being a two dimensional vector space and $\varphi$ a nonzero skew symmetric bilinear form on $U, \mathfrak{g}_{\overline{1}}=U \otimes_{F} U \otimes_{F} U$, with the natural multiplication in $\mathfrak{g}_{\overline{0}}$ and natural action of $\mathfrak{g}_{\overline{0}}$ on $\mathfrak{g}_{\overline{1}}$ in which the $i^{\text {th }}$ copy of $\mathfrak{s p}(U, \varphi)$ acts on the $i^{\text {th }}$ factor of $U$, and with the multiplication of odd elements given by:

$\left[u_{1} \otimes u_{2} \otimes u_{3}, v_{1} \otimes v_{2} \otimes v_{3}\right]=\varphi\left(u_{2}, v_{2}\right) \varphi\left(u_{3}, v_{3}\right) \varphi_{u_{1}, v_{1}}$

$$
+\alpha \varphi\left(u_{1}, v_{1}\right) \varphi\left(u_{3}, v_{3}\right) \varphi_{u_{2}, v_{2}}-(1+\alpha) \varphi\left(u_{1}, v_{1}\right) \varphi\left(u_{2}, v_{2}\right) \varphi_{u_{3}, v_{3}}
$$

for any $u_{i}, v_{i} \in U, i=1,2,3$. Here $\mathfrak{s}$ is the first copy of $\mathfrak{s p}(U, \varphi)$.

(vi) $\mathfrak{g}=G(3)$ and $\mathfrak{s}$ is the (unique) ideal of $\mathfrak{g}_{0}$ isomorphic to $\mathfrak{s l}(2)$.

(vii) $\mathfrak{g}=F(4)$ and $\mathfrak{s}$ is the (unique) ideal of $\mathfrak{g}_{0}$ isomorphic to $\mathfrak{s l}(2)$.

(viii) $\mathfrak{g}=\mathfrak{s p}(3,2 r), r \geq 1$, and $\mathfrak{s}$ is the copy of $\mathfrak{o}(3)$ in $\mathfrak{g}_{\overline{0}}$.

Moreover, different choose of the simple ideal $\mathfrak{s}$ in (ii) or (iv) give isomorphic pairs and two pairs in (v) corresponding to the values $\alpha_{1}$ and $\alpha_{2}$ are isomorphic if and only if either $\alpha_{1}=\alpha_{2}$ or $\alpha_{1}+\alpha_{2}=-1$.

Proof. A careful look at the list of simple Lie superalgebras in [11, Theorem 5] shows that the semisimple part of $\mathbf{W}(n)_{\overline{0}}(n \geq 2)$, of $\mathbf{S}(n)(n \geq 3)$ and of $\tilde{\mathbf{S}}(n)(n \geq 3)$, is isomorphic to $\mathfrak{s l}(n)$ [11, Propositions 3.1.1 and 3.3.1], while $\mathbf{W}(2)$ is isomorphic to $\mathfrak{s l}(1,2)$. Also, the semisimple part of $\mathbf{H}(n)(n \geq 4)$ is isomorphic to $o(n)[\mathbf{1 1}$, Proposition 3.3.6], while $\mathbf{H}(4)$ is isomorphic to $\mathfrak{p s l}(2,2)$. Hence, it is enough to deal with the classical algebras. One checks easily that the simple classical Lie superalgebras with $\mathfrak{g}_{0}$ containing a three dimensional simple ideal are those listed above. Since $\mathfrak{o s p}(4,2)$ is isomorphic to $D(2,1 ; 1)$, this has been excluded from (iii) and included in (v), and since $\mathfrak{s l}(1,2)$ is isomorphic to $\mathfrak{o} \mathfrak{s p}(2,2)$, this has been included in (iii).

The last assertion about cases (ii) and (iv) is clear. Also, the Lie algebras in (v) are the ones denoted by $\Gamma(1, \alpha,-(1+\alpha))$ in $[26$, p. 16-17]. Here we have three 
copies of $\mathfrak{s t}(2)$ in $\mathfrak{g}_{0}$, but there are isomorphisms preserving the three copies from $\Gamma\left(\sigma_{1}, \sigma_{2}, \sigma_{3}\right)\left(\sigma_{1}+\sigma_{2}+\sigma_{3}=0\right)$ onto $\Gamma\left(\eta \sigma_{1}, \eta \sigma_{2}, \eta \sigma_{3}\right)$ for any $0 \neq \eta \in F$, and also natural isomorphisms permuting the three copies of $\mathfrak{s l}(2)$ (and the corresponding $\sigma_{i}$ 's). Therefore, the distinguished copy of $\mathfrak{s l}(2)$ can always be taken to be the first one. Finally, if there is an isomorphism from $\Gamma(1, \alpha,-1-\alpha)$ onto $\Gamma(1, \beta,-1-\beta)$ that takes the first copy of $\mathfrak{s l}(2)$ in $\Gamma(1, \alpha,-1-\alpha)$ to the first copy of $\mathfrak{s l}(2)$ in $\Gamma(1, \beta,-1-\beta)$, then it takes the second copy of $\mathfrak{s l}(2)$ in $\Gamma(1, \alpha,-1-\alpha)$ to either the second or the third copy of $\mathfrak{s l}(2)$ in $\Gamma(1, \beta,-1-\beta)$, whence the last assertion of the Theorem.

Now we are ready for our main Theorem, it asserts that the examples in Section 3 exhaust all the simple $(-1,-1)$-BFKTS's:

THEOREM 4.3. Let $V$ be a finite dimensional simple $(-1,-1)$-BFKTS over a field $F$ of characteristic zero with associated symmetric bilinear form $\langle\mid\rangle$. Either:

(i) The multiplication in $V$ is given by

$$
x y z=\langle z \mid x\rangle y-\langle z \mid y\rangle x+\langle x \mid y\rangle z
$$

for any $x, y, z \in V$ (orthogonal type), or

(ii) There is a quadratic étale algebra $K$ over $F$ such that $V$ is a free $K$-module of rank at least 3, endowed with a hermitian form $h: V \times V \rightarrow K$ such that

$$
\left\{\begin{array}{l}
\langle x \mid y\rangle=\frac{1}{2}(h(x, y)+h(y, x)), \\
x y z=h(z, x) y-h(z, y) x+h(x, y) z
\end{array}\right.
$$

for any $x, y, z \in V$ (unitarian type).

(iii) There is a quaternion algebra $Q$ over $F$ such that $V$ is a free left $Q$-module of rank $\geq 2$, endowed with a hermitian form $h: V \times V \rightarrow Q$ such that

$$
\left\{\begin{array}{l}
\langle x \mid y\rangle=\frac{1}{2}(h(x, y)+h(y, x)) \\
x y z=h(z, x) y-h(z, y) x+h(x, y) z
\end{array}\right.
$$

for any $x, y, z \in V$ (symplectic type).

(iv) $\operatorname{dim}_{F} V=4$ and there is a nonzero skew symmetric multilinear form $\Phi: V \times$ $V \times V \times V \rightarrow F$ such that for any $x, y, z \in V$ :

$$
x y z=[x y z]+\langle z \mid x\rangle y-\langle z \mid y\rangle x+\langle x \mid y\rangle z
$$

where $[x y z]$ is defined by means of $\Phi(x, y, z, t)=\langle[x y z] \mid t\rangle$ for any $x, y, z, t \in V$. In this case, there is a nonzero scalar $\mu \in F$ such that (3.8) holds $\left(D_{\mu}\right.$-type).

(v) $\operatorname{dim}_{F} V=7$ and there is an eight dimensional Cayley-Dickson algebra $C$ over $F$ with trace $t$ and a nonzero scalar $\alpha \in F$ such that $V=C_{0}=\{x \in C: t(x)=0\}$, and for any $x, y, z \in V$ :

$$
\left\{\begin{array}{l}
\langle x \mid y\rangle=-2 \alpha t(x y) \\
x y z=\alpha\left(D_{x, y}(z)-2 t(x y) z\right)
\end{array}\right.
$$

where $D_{x, y}$ is the inner derivation of $C$ given by (3.11) (G-type). 
(vi) $\operatorname{dim}_{F} V=8$ and $(V,\langle\mid\rangle)$ is endowed with a 3-fold vector cross product $X$ of type I such that

$$
x y z=\frac{1}{3} X(x, y, z)+\langle z \mid x\rangle y-\langle z \mid y\rangle x+\langle x \mid y\rangle z
$$

for any $x, y, z \in V$. (F-type.)

Moreover, two triple systems in different items cannot be isomorphic and:

(i') Two triple systems of orthogonal type are isomorphic if and only if the corresponding symmetric bilinear forms are isometric.

(ii') Two triple systems of unitarian type $V_{1}$ and $V_{2}$, with associated quadratic étale algebras $K_{1}$ and $K_{2}$ and hermitian forms $h_{1}$ and $h_{2}$, are isomorphic if and only if the hermitian pairs $\left(V_{1}, h_{1}\right)$ and $\left(V_{2}, h_{2}\right)$ are isomorphic; that is, there is an isomorphism of $F$-algebras $\sigma: K_{1} \rightarrow K_{2}$ and a linear bijection $\varphi: V_{1} \rightarrow V_{2}$ such that $h_{2}(\varphi(x), \varphi(y))=$ $\sigma\left(h_{1}(x, y)\right)$ for any $x, y \in V_{1}$.

(iii') Two triple systems of symplectic type $V_{1}$ and $V_{2}$, with associated quaternion algebras $Q_{1}$ and $Q_{2}$ and hermitian forms $h_{1}$ and $h_{2}$, are isomorphic if and only if the hermitian pairs $\left(V_{1}, h_{1}\right)$ and $\left(V_{2}, h_{2}\right)$ are isomorphic.

(iv') Two triple systems of $D_{\mu}$-type, with associated scalars $\mu_{1}$ and $\mu_{2}$, are isomorphic if and only if the corresponding symmetric bilinear forms are isometric and $\mu_{1}=\mu_{2}$.

(v') Two triple systems of $G$-type, with associated Cayley-Dickson algebras $C_{1}$ and $C_{2}$ and scalars $\alpha_{1}$ and $\alpha_{2}$, are isomorphic if and only if so are $C_{1}$ and $C_{2}$ and $\alpha_{1}=\alpha_{2} \gamma^{2}$ for some $0 \neq \gamma \in F$.

(vi') Two triple systems of F-type $V_{1}$ and $V_{2}$, with associated type I 3-fold vector cross products $X_{1}$ and $X_{2}$, are isomorphic if and only if so are the triple systems $\left(V_{1}, X_{1}\right)$ and $\left(V_{2}, X_{2}\right)$.

Proof. First, the new triple product defined on $V$ by $[x y z]=x y z-\langle z \mid x\rangle y+$ $\langle z \mid y\rangle x-\langle x \mid y\rangle z$ for any $x, y, z \in V$ is skew symmetric because of (2.5a). If this is identically zero, $V$ is of orthogonal type. Otherwise, if the dimension of $V$ is $4, V$ is of $\mathrm{D}_{\mu}$-type.

Hence, in what follows, assume that $\operatorname{dim}_{F} V \neq 4$. Then, after extending scalars to an algebraic closure $\bar{F}$ of $F$, if $\bar{V}=\bar{F} \otimes_{F} V,(\mathfrak{g}(\bar{V}), \mathfrak{s}(\bar{V}))$ is one of the pairs considered in cases (i), (iii), (iv), (vi) or (vii) in Theorem 4.2. Note that case (viii) does not appear since there $\mathfrak{g}_{\overline{1}}$ is a direct sum of adjoint modules for $\mathfrak{s}$ instead of a direct sum of two dimensional irreducible modules.

Because of Theorem 4.1 and the computations in Section 3, and since the classical Lie superalgebras other than $D(2,1 ; \alpha)$ 's are determined by its even part and the structure of $\mathfrak{g}_{\overline{1}}$ as a $\mathfrak{g}_{\overline{0}}$-module [11, Proposition 2.1.4], it follows that case (i) in Theorem 4.2 corresponds to the unitarian type with $\bar{K}=\bar{F} \times \bar{F}$ and $\operatorname{dim}_{F} V \geq 6$, case (iii) in 4.2 corresponds to the orthogonal type, case (iv) to the symplectic type and $\operatorname{dim}_{F} V \geq 8$ and cases (vi) and (vii) to $\mathrm{G}$ and $\mathrm{F}$ types.

Therefore, it is enough to deal with the forms over $F$ of the simple $(-1,-1)$ BFKTS's over $\bar{F}$ considered in Section 3 with dimension $\neq 4$.

It is clear that if $\bar{V}$ is of orthogonal type, so is $V$. If $\bar{V}$ is of unitarian type with $\operatorname{dim}_{F} V \geq 6$, then since $\bar{K}=\operatorname{End}_{\mathfrak{d}}(\bar{V})=\bar{F} \otimes_{F} \operatorname{End}_{\mathfrak{d}}(V), K=\operatorname{End}_{\mathfrak{d}}(V)$ is a quadratic étale algebra over $F$; besides, there is a $\bar{K}$-hermitian form $\bar{h}: \bar{V} \times \bar{V} \rightarrow \bar{K}$ such that $x y z=\bar{h}(z, x) y-\bar{h}(z, y) x+\bar{h}(x, y) z$ for any $x, y, z \in \bar{V}$. But if $\{1, i\}$ is an $F$-basis of $K$ with $i^{2}=\alpha \in F$, then $\bar{h}(x, y)=\langle x \mid y\rangle-\alpha^{-1}\langle x \mid i y\rangle i$ for any $x, y \in \bar{V}$. Since both 
$\langle x \mid y\rangle$ and $\langle x \mid i y\rangle$ are in $F$ in case $x, y \in V$, it follows that $\bar{h}$ restricts to an hermitian form $h: V \times V \rightarrow K$ and $V$ is the corresponding simple $(-1,-1)$-BFKTS of unitarian type. A similar argument works in case $\bar{V}$ is of symplectic type and $\operatorname{dim}_{F} V \geq 8$. In this case $\overline{\mathfrak{d}}=\overline{\mathfrak{b}} \oplus \overline{\mathfrak{s}}$ with $\overline{\mathfrak{s}} \cong \mathfrak{s l}(2, \bar{F}) \not \overline{\mathfrak{b}}$, so that $\mathfrak{d}=\mathfrak{b} \oplus \mathfrak{s}$ for a suitable unique ideal $\mathfrak{b}$ and $\bar{Q}=\operatorname{End}_{\bar{b}}(\bar{V})=\bar{F} \otimes_{F} \operatorname{End}_{\mathfrak{b}}(V)$. Hence $\operatorname{End}_{\mathfrak{b}}(V)=Q$ is a quaternion algebra and $V$ is a free $Q$-module. Now one takes a suitable $F$-basis $\{1, i, j, k\}$ of $Q$ and argues as above.

If $\bar{V}$ is of G-type, then $\mathfrak{d}$ is a form of $G_{2}$, so there is an eight-dimensional CayleyDickson algebra $C$ over $F$ such that $\mathfrak{d} \cong \operatorname{Der} C$ and $V$ is, up to isomorphism, its seven dimensional irreducible module for $\mathfrak{d}$, that is $C_{0}$, the set of traceless elements in $C$. Since $\operatorname{Hom}_{\mathfrak{d}}\left(V \otimes_{F} V, \mathfrak{d}\right)$ is one-dimensional, after identifying $V$ with $C_{0}$ there exists a nonzero $\alpha \in F$ such that $d_{x, y}=\alpha D_{x, y}$ for any $x, y \in C_{0}=V$. From here, using (2.3c), it follows that $V$ is of G-type.

Finally, if $\bar{V}$ is of $F$-type, define $X: V \times V \times V \rightarrow F$ by $X(x, y, z)=3(x y z-$ $\langle z \mid x\rangle y+\langle z \mid y\rangle x-\langle x \mid y\rangle z)$, for any $x, y, z \in V$. Then $X$ is a 3-fold vector cross product of type I (because it is so after extending scalars) and hence $V$ is of F-type.

Moreover, two simple $(-1,-1)$-BFKTS's of different types cannot be isomorphic because the corresponding Lie algebras of inner derivations are not. Also note that, because of (2.5a), any isomorphism among two $(-1,-1)$-BFKTS's is an isometry of the corresponding symmetric bilinear forms. Now (i') is clear and (ii') (respectively (iii')) follows from the fact that $K_{1}$ and $K_{2}$ (resp. $Q_{1}$ and $Q_{2}$ ) are determined as centralizers of the action of a suitable ideal of the Lie algebra of inner derivations.

Let us check $\left(\mathrm{iv}^{\prime}\right)$, so let $\left(V_{i},(x y z)_{i}\right)$ be two simple $(-1,-1)$-BFKTS's of $\mathrm{D}_{\mu_{i}}$ type $(i=1,2)$. If $\varphi: V_{1} \rightarrow V_{2}$ is an isomorphism, then it is an isometry and thus $\varphi\left([x y z]_{1}\right)=[\varphi(x) \varphi(y) \varphi(z)]_{2}$ for any $x, y, z \in V_{1}$. Hence

$$
\left\langle\varphi\left(\left[x_{1} x_{2} x_{3}\right]_{1}\right) \mid \varphi\left(\left[x_{1} x_{2} x_{3}\right]_{1}\right)\right\rangle_{2}=\left\langle\left[x_{1} x_{2} x_{3}\right]_{1} \mid\left[x_{1} x_{2} x_{3}\right]_{1}\right\rangle_{1}=\mu_{1} \operatorname{det}\left(\left\langle x_{i} \mid x_{j}\right\rangle_{1}\right),
$$

but also

$$
\begin{aligned}
\left\langle\varphi\left(\left[x_{1} x_{2} x_{3}\right]_{1}\right) \mid \varphi\left(\left[x_{1} x_{2} x_{3}\right]_{1}\right)\right\rangle_{2} & =\left\langle\left[\varphi\left(x_{1}\right) \varphi\left(x_{2}\right) \varphi\left(x_{3}\right)\right]_{2} \mid\left[\varphi\left(x_{1}\right) \varphi\left(x_{2}\right) \varphi\left(x_{3}\right)\right]_{2}\right\rangle_{2} \\
& =\mu_{2} \operatorname{det}\left(\left\langle\varphi\left(x_{i}\right) \mid \varphi\left(x_{j}\right)\right\rangle_{2}\right) \\
& =\mu_{2} \operatorname{det}\left(\left\langle x_{i} \mid x_{j}\right\rangle_{1}\right) .
\end{aligned}
$$

Therefore, $\mu_{1}=\mu_{2}$. Conversely, assume that $\varphi: V_{1} \rightarrow V_{2}$ is an isometry and that $\mu_{1}=$ $\mu_{2}=\mu$. Consider $\Phi_{i}: V_{i}^{4} \rightarrow F(i=1,2)$ given by $\Phi_{i}\left(x_{1}, x_{2}, x_{3}, x_{4}\right)=\left\langle\left[x_{1} x_{2} x_{3}\right]_{i} \mid x_{4}\right\rangle_{i}$. Also, let $\tilde{\Phi}_{1}: V_{1}^{4} \rightarrow F$ be defined by

$$
\tilde{\Phi}_{1}\left(x_{1}, x_{2}, x_{3}, x_{4}\right)=\Phi_{2}\left(\varphi\left(x_{1}\right), \varphi\left(x_{2}\right), \varphi\left(x_{3}\right), \varphi\left(x_{4}\right)\right)
$$

for any $x_{1}, x_{2}, x_{3}, x_{4} \in F$. Since $\operatorname{dim}_{F} V_{1}=4$ and both $\Phi_{1}$ and $\tilde{\Phi}_{1}$ are skew symmetric, they are proportional, and hence there is a nonzero scalar $\alpha \in F$ such that $\tilde{\Phi}_{1}=\alpha \Phi_{1}$. For any $x_{1}, x_{2}, x_{3}, y_{1}, y_{2}, y_{3} \in F$ :

$$
\begin{aligned}
\Phi_{2}\left(\varphi\left(x_{1}\right), \varphi\left(x_{2}\right), \varphi\left(x_{3}\right), \varphi\left(\left[y_{1} y_{2} y_{3}\right]_{1}\right)\right) & =\tilde{\Phi}_{1}\left(x_{1}, x_{2}, x_{3},\left[y_{1} y_{2} y_{3}\right]_{1}\right) \\
& =\alpha \Phi_{1}\left(x_{1}, x_{2}, x_{3},\left[y_{1} y_{2} y_{3}\right]_{1}\right) \\
& =\alpha \mu \operatorname{det}\left(\left\langle x_{i} \mid y_{j}\right\rangle_{1}\right) \\
& =\alpha \mu \operatorname{det}\left(\left\langle\varphi\left(x_{i}\right) \mid \varphi\left(y_{j}\right)\right\rangle_{2}\right) \\
& =\Phi_{2}\left(\varphi\left(x_{1}\right), \varphi\left(x_{2}\right) \varphi\left(x_{3}\right), \alpha\left[\varphi\left(y_{1}\right) \varphi\left(y_{2}\right) \varphi\left(y_{3}\right)\right]_{2}\right),
\end{aligned}
$$


where we have used (3.8) and the fact that $\varphi$ is an isometry. Thus $\varphi\left(\left[y_{1} y_{2} y_{3}\right]_{1}\right)=$ $\alpha\left[\varphi\left(y_{1}\right) \varphi\left(y_{2}\right) \varphi\left(y_{3}\right)\right]_{2}$ for any $y_{1}, y_{2}, y_{3} \in V_{1}$. But now, again by (3.8), this shows that $\mu \operatorname{det}\left(\left\langle y_{i} \mid y_{j}\right\rangle_{1}\right)=\alpha^{2} \mu \operatorname{det}\left(\left\langle y_{i} \mid y_{j}\right\rangle_{1}\right)$ for any $y_{i}$ 's, so that $\alpha^{2}=1$. If $\alpha=1$ we are done, otherwise $\alpha=-1$. In this latter case, choose any isometry $\sigma$ of $\langle\mid\rangle_{1}$ with $\operatorname{det} \sigma=-1$ and consider $\hat{\varphi}=\varphi \sigma: V_{1} \rightarrow V_{2}$. Then if $\hat{\Phi}_{1}\left(x_{1}, x_{2}, x_{3}, x_{4}\right)=$ $\Phi_{2}\left(\hat{\varphi}\left(x_{1}\right), \hat{\varphi}\left(x_{2}\right), \hat{\varphi}\left(x_{3}\right), \hat{\varphi}\left(x_{4}\right)\right)$ for any $x_{i} \in V_{1}(i=1,2,3,4)$, we have:

$$
\begin{aligned}
\hat{\Phi}_{1}\left(x_{1}, x_{2}, x_{3}, x_{4}\right) & =\Phi_{2}\left(\hat{\varphi}\left(x_{1}\right), \hat{\varphi}\left(x_{2}\right), \hat{\varphi}\left(x_{3}\right), \hat{\varphi}\left(x_{4}\right)\right) \\
=\tilde{\Phi}_{1}\left(\sigma\left(x_{1}\right), \sigma\left(x_{2}\right), \sigma\left(x_{3}\right), \sigma\left(x_{4}\right)\right) & =\alpha(\operatorname{det} \sigma) \Phi_{1}\left(x_{1}, x_{2}, x_{3}, x_{4}\right)=\Phi_{1}\left(x_{1}, x_{2}, x_{3}, x_{4}\right),
\end{aligned}
$$

because $\alpha=-1=\operatorname{det} \sigma$ and $\Phi_{1}$ is multilinear and alternating. The same argument as above, with $\tilde{\Phi}_{1}$ replaced by $\hat{\Phi}_{1}$ shows that $\hat{\varphi}$ is an isomorphism between the two triple systems.

With regard to $\left(\mathrm{v}^{\prime}\right)$, if $\varphi: V^{1} \rightarrow V^{2}$ is an isomorphism of two triple systems of G-type with associated Cayley-Dickson algebras $C^{1}$ and $C^{2}$ and scalars $\alpha_{1}$ and $\alpha_{2}$, then $\varphi$ is an isometry of the associated symmetric bilinear forms and for any $x, y, z \in V_{1}$

$$
\varphi\left(d_{x, y} z\right)=d_{\varphi(x), \varphi(y)} \varphi(z)
$$

Also, $\phi: \mathfrak{d}^{1}=d_{V^{1}, V^{1}} \rightarrow \mathfrak{d}^{2}: d \mapsto \varphi d \varphi^{-1}$ is an isomorphism of Lie algebras and $\varphi$ becomes an isomorphism of $\mathfrak{d}^{1}$-modules, where $V^{2}$ is a $\mathfrak{d}^{1}$-module through $\phi$. Since $\operatorname{Hom}_{\mathfrak{d}^{1}}\left(\Lambda^{2}\left(V^{1}\right), V^{1}\right)$ is spanned by $x \wedge y \mapsto[x, y]=x y-y x$ (multiplication in $C^{1}$ ), there is a nonzero scalar $\mu \in F$ such that

$$
\varphi([x, y])=\mu[\varphi(x), \varphi(y)]
$$

for any $x, y \in V^{1}=C_{0}^{1}=\left\{z \in C^{1}: t(z)=0\right\}$. In particular, $\mu \varphi:\left(C_{0}^{1},[],\right) \rightarrow\left(C_{0}^{2},[],\right)$ is an isomorphism of Malcev algebras and hence $C^{1}$ and $C^{2}$ are isomorphic (see, for instance, $[3,(3.1)])$. But the associator $(x, y, z)=(x y) z-x(y z)$ in $C^{1}$ is skew symmetric on its arguments, so for any $x, y, z \in C^{1},(x, y, z)=-(x, z, y)=(z, x, y)=(y, z, x)$, so that $L_{x y}-L_{x} L_{y}=\left[L_{x}, R_{y}\right]=R_{y} R_{x}-R_{x y}=\left[R_{x}, L_{y}\right]$, hence $a d_{x y}-L_{x} L_{y}+R_{y} R_{x}=$ $2\left[L_{x}, R_{y}\right]$ for any $x, y \in C^{1}$, where $\operatorname{ad}_{x} y=[x, y]=\left(L_{x}-R_{x}\right)(y)$. Permuting $x$ and $y$ and subtracting we get $\operatorname{ad}_{[x, y]}=\left[L_{x}, L_{y}\right]+\left[R_{x}, R_{y}\right]+4\left[L_{x}, R_{y}\right]=D_{x, y}+3\left[L_{x}, R_{y}\right]$. On the other hand,

$$
\begin{aligned}
{\left[\operatorname{ad}_{x}, \mathrm{ad}_{y}\right] } & =\left[L_{x}-R_{x}, L_{y}-R_{y}\right]=\left[L_{x}, L_{y}\right]+\left[R_{x}, R_{y}\right]-2\left[L_{x}, R_{y}\right] \\
& =D_{x, y}-3\left[L_{x}, R_{y}\right]
\end{aligned}
$$

and from here we conclude that $2 D_{x, y}=\operatorname{ad}_{[x, y]}+\left[\operatorname{ad}_{x}, \operatorname{ad}_{y}\right]$ for any $x, y \in C^{1}$. Since $d_{x, y}=\alpha_{1} D_{x, y}$ and $d_{\varphi(x), \varphi(y)}=\alpha_{2} D_{\varphi(x), \varphi(y)}$ for any $x, y \in V^{1}$, equation (4.3) gives:

$$
\begin{aligned}
\varphi d_{x, y} & =\frac{\alpha_{1}}{2} \varphi\left(\operatorname{ad}_{[x, y]}+\left[\operatorname{ad}_{x}, \operatorname{ad}_{y}\right]\right)=\frac{\alpha_{1}}{2} \mu^{2}\left(\operatorname{ad}_{[\varphi(x), \varphi(y)]}+\left[\operatorname{ad}_{\varphi(x)}, \operatorname{ad}_{\varphi(y)}\right]\right) \varphi \\
& =\frac{\alpha_{1}}{2} \mu^{2} D_{\varphi(x), \varphi(y)} \varphi
\end{aligned}
$$

while $d_{\varphi(x), \varphi(y)}=\frac{\alpha_{2}}{2} D_{\varphi(x), \varphi(y)}$ for any $x, y \in V^{1}$, so that equation (4.2) gives $\alpha_{1} \mu^{2}=\alpha_{2}$, as desired. Conversely, if $\psi: C^{1} \rightarrow C^{2}$ is an isomorphism and $\alpha_{2}=\alpha_{1} \mu^{2}$, then the map $\varphi: V^{1}=C_{0}^{1} \rightarrow V^{2}=C_{0}^{2}$, given by $\varphi(x)=\mu^{-1} \psi(x)$ for any $x \in V^{1}$, is an isomorphism of triple systems. 
We are left with the isomorphism problem for the $(-1,-1)$-BFKTS's of F-type. For these we need some preliminaries, which have their own independent interest:

LEMMA 4.4. Let $X$ be a 3-fold vector cross product of type I on an eight dimensional vector space $V$ over a field $F$ of characteristic $\neq 2$, and let $\langle\mid\rangle$ be the associated nondegenerate symmetric bilinear form (so that (3.11) is satisfied). Then $\langle\mid\rangle$ is determined by $X$.

Proof. Because of (3.12), for any $a, b, c, d \in V$ :

$$
\begin{aligned}
& -\langle d \mid X(a, b, X(a, b, c))\rangle=\langle X(a, b, c) \mid X(a, b, d)\rangle=\left|\begin{array}{ccc}
\langle a \mid a\rangle & \langle a \mid b\rangle & \langle a \mid d\rangle \\
\langle b \mid a\rangle & \langle b \mid b\rangle & \langle b \mid d\rangle \\
\langle c \mid a\rangle & \langle c \mid b\rangle & \langle c \mid d\rangle
\end{array}\right| \\
& =\langle\langle a \wedge b \mid a \wedge b\rangle c-\langle a \wedge b \mid a \wedge c\rangle b+\langle a \wedge b \mid b \wedge c\rangle a \mid d\rangle
\end{aligned}
$$

where $\langle a \wedge b \mid u \wedge v\rangle=\left|\begin{array}{ll}\langle a \mid u\rangle & \langle a \mid v\rangle \\ \langle b \mid u\rangle & \langle b \mid v\rangle\end{array}\right|$ for any $a, b, u, v \in V$. By nondegeneracy of $\langle\mid\rangle$, this gives:

$$
X(a, b, X(a, b, c))=\langle a \wedge b \mid c \wedge b\rangle a+\langle a \wedge b \mid a \wedge c\rangle b-\langle a \wedge b \mid a \wedge b\rangle c .
$$

Hence, for any $a, b, c \in V$, if $d=X(a, b, c)$, then $X(a, b, d) \in F a+F b+F c$ and, similarly ( since $d=X(b, c, a)=X(c, a, b)), X(b, c, d), X(a, c, d) \in F a+F b+F c$, so that $W=F a+F b+F c+F d$ is closed under $X$. Let us prove now that for any $0 \neq v \in V$ :

$$
X(v, V, V)=\{x \in V:\langle v \mid x\rangle=0\}
$$

Because of (3.12), $X(v, V, V) \subseteq\{x \in V:\langle x \mid v\rangle=0\}$. Now, take $a=v$ and let $b \in V$ linearly independent with $a$ and such that $\langle\mid\rangle$ is nondegenerate on $W_{b}=F a+F b$. By (4.4) $c \in X(a, b, V) \subseteq X(v, V, V)$ for any $c \in W_{b}^{\perp}=\{x \in V:\langle x \mid a\rangle=0=\langle x \mid b\rangle\}$. Take any two such $b$ 's with different $W_{b}$ 's, then the sum of the $W_{b}^{\perp}$ 's is $\{x \in V$ : $\langle x \mid v\rangle=0\}$, so (4.5) follows.

Thus, assume that $X$ is also a 3 -fold vector cross product of type I relative to another nondegenerate symmetric bilinear form ( | ) on $V$. Then for any $0 \neq u, v \in V$, if $\langle u \mid v\rangle=0$, then $u \in X(v, V, V)$ by (4.5), so by (3.12), also $(u \mid v)=0$. The only possibility then is that $(\mid)=\alpha\langle\mid\rangle$ for some nonzero scalar $\alpha \in F$. But then (3.12) implies that $\alpha^{3}=\alpha$, so $\alpha= \pm 1$, and (3.13) that $\alpha=1$.

Note that if $X$ is a 3 -fold vector cross product of type I on an eight dimensional vector space $V$ relative to the nondegenerate symmetric bilinear form $\langle\mid\rangle$, then $X$ is a 3 -fold vector cross product of type II relative to $-\langle\mid\rangle$. Also note that $\langle\mid\rangle$ does not determine $X$, since not every orthogonal transformation relative to $\langle\mid\rangle$ is an automorphism of $X([4])$.

COROLLARY 4.5. Let $X_{i}$ be a 3-fold vector cross product on an eight dimensional vector space $V_{i}$ over a field $F$ of characteristic $\neq 2$ with associated nondegenerate symmetric bilinear form $\langle\mid\rangle_{i}(i=1,2)$. Then if $\varphi:\left(V_{1}, X_{1}\right) \rightarrow\left(V_{2}, X_{2}\right)$ is an isomorphism, then it is also an isometry $\varphi:\left(V_{1},\langle\mid\rangle_{1}\right) \rightarrow\left(V_{2},\langle\mid\rangle_{2}\right)$.

Now, the proof of item ( $\left.\mathrm{vi}^{\prime}\right)$ in Theorem 4.3 follows immediately from the Corollary above and this finishes its proof. 


\section{REFERENCES}

1. P. Alberca Bjerregaard, A. Elduque, C. Martín González and F. J. Navarro Márquez, On the Cartan-Jacobson Theorem, J. Algebra 250 (2002), no. 2, 397-407.

2. G. Benkart and A. Elduque, The Tits construction and the exceptional simple classical Lie superalgebras, Quart. J. Math. Oxford, to appear.

3. A. Elduque, On maximal subalgebras of central simple Mal'cev algebras, J. Algebra 103 (1986), no. 1, 216-227.

4. A. Elduque, On a class of ternary composition algebras, J. Korean Math. Soc. 33 (1996), no. $1,183-203$.

5. A. Elduque, On triality and automorphisms and derivations of composition algebras, Linear Algebra Appl. 314 (2000), no. 1-3, 49-74.

6. A. Elduque, Quaternion, octonions, and the forms of the exceptional simple classical Lie superalgebras, submitted. Jordan Theory Preprint Archives: http://mathematik.uibk.ac.at/ jordan/ (paper no. 116).

7. A. Elduque and H. C. Myung, The reductive pair $\left(B_{3}, G_{2}\right)$ and affine connections on $S^{7}$, J. Pure Appl. Algebra 86 (1993), no. 2, 155-171.

8. J. R. Faulkner and J. C. Ferrar, On the structure of symplectic ternary algebras, Nederl. Akad. Wetensch. Proc. Ser. A 75=Indag. Math. 34 (1972), 247-256.

9. J. R. Faulkner and J. C. Ferrar, Simple anti-Jordan pairs, Commun. Algebra 8 (1980), no. $11,993-1013$.

10. H. Freudenthal, Beziehungen der $E_{7}$ und $E_{8}$ zur Oktavenebene. I, II, Nederl. Akad. Wetensch. Proc. Ser. A. $57=$ Indag. Math. 16 (1954), 218-230 and 363-380.

11. V. G. Kac, Lie superalgebras, Adv. Math. 26 (1977), no. 1, 8-96.

12. N. Kamiya, A structure theory of Freudenthal-Kantor triple systems, J. Algebra 110 (1987), no. 1, 108-123.

13. N. Kamiya, A structure theory of Freudenthal-Kantor triple systems II, Comment. Math. Univ. St. Paul. 38 (1989), no. 1, 41-60.

14. N. Kamiya, A structure theory of Freudenthal-Kantor triple systems. III, Mem. Fac. Sci. Shimane Univ. 23 (1989), 33-51.

15. N. Kamiya, The construction of all simple Lie algebras over $\mathbf{C}$ from balanced Freudenthal-Kantor triple systems, in Contributions to general algebra, 7 (Vienna, 1990) (HölderPichler-Tempsky, Vienna, 1991), 205-213.

16. N. Kamiya, On Freudenthal-Kantor triple systems and generalized structurable algebras, in Non-associative algebra and its applications (Oviedo, 1993), Math. Appl. 303 (Kluwer, Dordrecht, 1994), 198-203.

17. N. Kamiya, On the Peirce decompositions for Freudenthal-Kantor triple systems, Commun. Algebra 25 (1997), no. 6, 1833-1844.

18. N. Kamiya and S. Okubo, On $\delta$-Lie supertriple systems associated with $(\epsilon, \delta)$ Freudenthal-Kantor supertriple systems, Proc. Edinburgh Math. Soc. (2) 43 (2000), no. 2, 243-260.

19. N. Kamiya and S. Okubo, Construction of Lie superalgebras $D(2,1 ; \alpha), G(3)$ and $F(4)$ from some triple systems, Proc. Edinburgh Math. Soc. (2) 46 (2003), 87-98.

20. I. L. Kantor, Models of the exceptional Lie algebras (Russian), Dokl. Akad. Nauk SSSR 208 (1973), no. 6, 1276-1279. English translation in Soviet Math. Dokl. 14, no. 1, 254-258.

21. K. Meyberg, Eine Theorie der Freudenthalschen Tripelsysteme. I, II, Nederl. Akad. Wetensch. Proc. Ser. A 71 = Indag. Math. 30 (1968), 162-174 and 175-190.

22. S. Okubo, Triple products and Yang-Baxter equation. I. Octonionic and quaternionic triple systems, J. Math. Phys. 34 (1993), no. 7, 3273-3291.

23. S. Okubo Introduction to octonion and other non-associative algebras in physics, Montroll Memorial Lecture Series in Mathematical Physics No. 2 (Cambridge University Press, 1995).

24. S. Okubo and N. Kamiya, Quasi-classical Lie superalgebras and Lie super triple systems, Commun. Algebra 30 (2002), no. 8, 3825-3850.

25. R. D. Schafer, An introduction to nonassociative algebras, Pure and Applied Mathematics, Vol. 22 (Academic Press, 1966).

26. M. Scheunert, The theory of Lie superalgebras. An introduction, Lecture Notes in Mathematics No 716 (Springer-Verlag, 1979). 
27. K. Yamaguti, Constructions of Lie (super)algebras from Freudenthal-Kantor triple system $U(\epsilon, \delta)$, Proceedings of the 14th ICGTMP, Seoul, 1985 (World Sci. Publishing, Singapore, 1986), 222-225.

28. K. Yamaguti and H. Asano, On the Freudenthal's construction of exceptional Lie algebras, Proc. Japan Acad. 51 (1975), no. 4, 253-258.

29. K. Yamaguti and A. Ono, On representations of Freudenthal-Kantor triple systems $U(\epsilon, \delta)$, Bull. Fac. School Ed. Hiroshima Univ. Part II 7 (1984), 43-51. 